\title{
Pain perception in Parkinson's disease: A systematic review and meta-analysis of experimental studies
}

\author{
Trevor Thompson ${ }^{\mathrm{a}, *}$, Katy Gallop ${ }^{\mathrm{b}}$, Christoph U. Correll ${ }^{\mathrm{c}, \mathrm{d}}$, Andre F. Carvalho ${ }^{\mathrm{e}}$, \\ Nicola Veronese ${ }^{\mathrm{f}}$, Ellen Wright ${ }^{\mathrm{g}}$, Brendon Stubbs ${ }^{\mathrm{h}, \mathrm{i}}$ \\ a Faculty of Education and Health, University of Greenwich, London SE9 2UG, UK \\ ${ }^{\mathrm{b}}$ Acaster Consulting, London SE3 7HU, UK \\ c epartment of Psychiatry, The Zucker Hillside Hospital, Northwell Health, Glen Oaks, NY 11004, USA \\ d Department of Psychiatry and Molecular Medicine, Hofstra Northwell School of Medicine, Hempstead, NY 11549, USA \\ e Department of Clinical Medicine and Translational Psychiatry Research Group, Faculty of Medicine, Federal University of Ceará, Fortaleza, CE, Brazil \\ ${ }^{\mathrm{f}}$ Institute for Clinical Research and Education in Medicine, I.R.E.M., Padova, Italy \\ g Department of Primary Care and Public Health Sciences, King's College London, London SE1 3QD, UK \\ h Physiotherapy Department, South London and Maudsley NHS Foundation Trust, London SE5 8AZ, UK \\ ${ }^{\mathrm{i}}$ Health Service and Population Research Department, King's College London, London SE5 8AF, UK
}

\section{A R T I C L E I N F O}

\section{Article history:}

Received 19 December 2016

Received in revised form 25 January 2017

Accepted 25 January 2017

Available online 4 February 2017

\section{Keywords:}

Parkinson's disease

Dopamine

Pain

Meta-analysis

Systematic review

\begin{abstract}
A B S T R A C T
While hyperalgesia (increased pain sensitivity) has been suggested to contribute to the increased prevalence of clinical pain in Parkinson's disease (PD), experimental research is equivocal and mechanisms are poorly understood. We conducted a meta-analysis of studies comparing PD patients to healthy controls (HCs) in their response to experimental pain stimuli. Articles were acquired through systematic searches of major databases from inception until 10/2016. Twenty-six studies met inclusion criteria, comprising 1292 participants ( $\mathrm{PD}=739, \mathrm{HCs}=553$ ). Random effects meta-analysis of standardized mean differences $(S M D)$ revealed lower pain threshold (indicating hyperalgesia) in PD patients during unmedicated OFF states $(S M D=0.51)$ which was attenuated during dopamine-medicated ON states $(S M D=0.23)$, but unaffected by age, PD duration or PD severity. Analysis of 6 studies employing suprathreshold stimulation paradigms indicated greater pain in PD patients, just failing to reach significance $(S M D=0.30$, $p=0.06$ ). These findings (a) support the existence of hyperalgesia in PD, which could contribute to the onset/intensity of clinical pain, and (b) implicate dopamine deficiency as a potential underlying mechanism, which may present opportunities for the development of novel analgesic strategies.
\end{abstract}

(C) 2017 Elsevier B.V. All rights reserved.

\section{Contents}

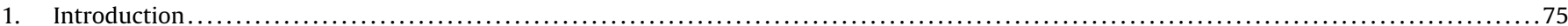

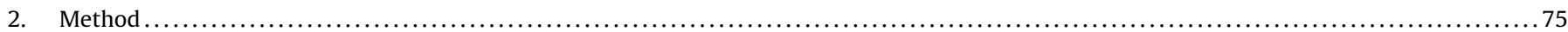

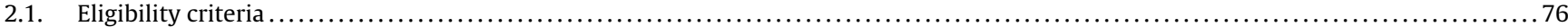

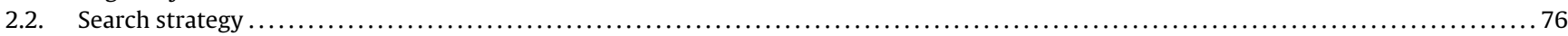

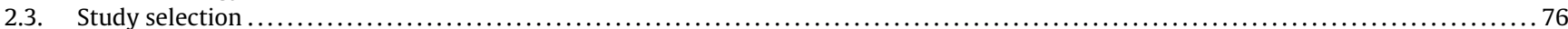

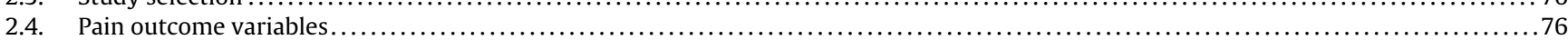

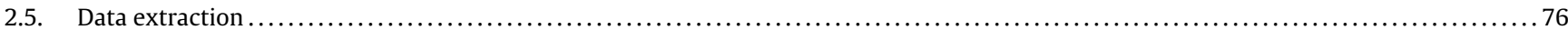

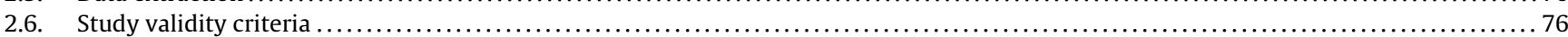

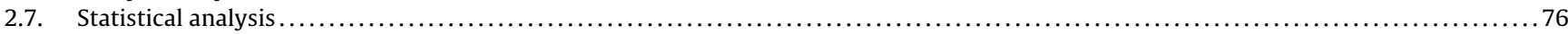

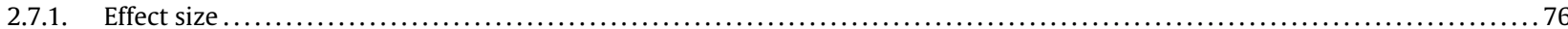

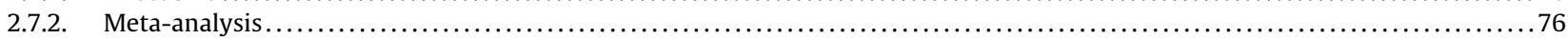

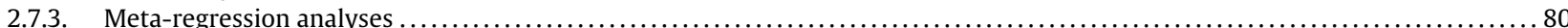

\footnotetext{
* Corresponding author.

E-mail address: t.thompson@gre.ac.uk (T. Thompson).
} 


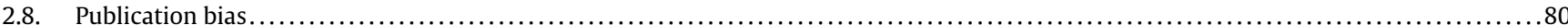

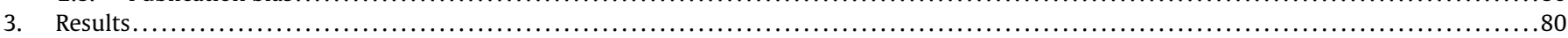

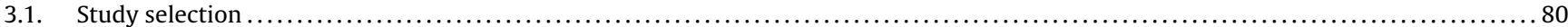

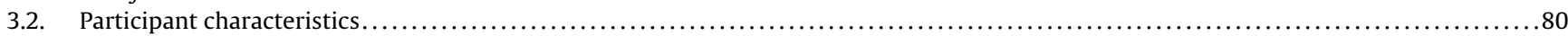

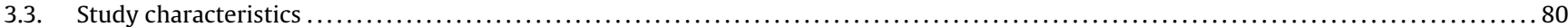

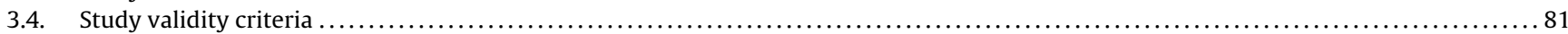

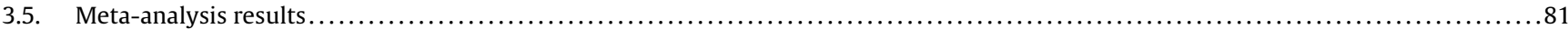

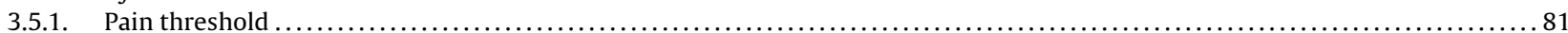

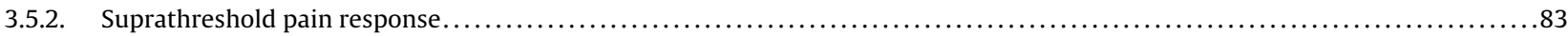

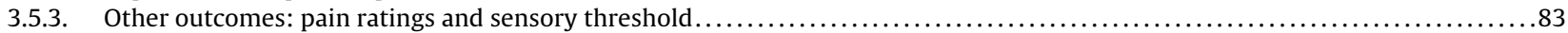

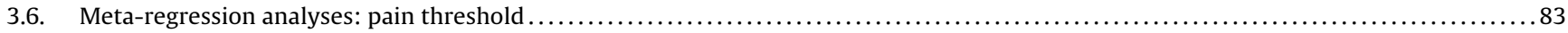

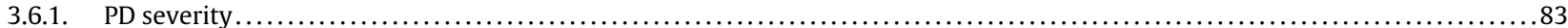

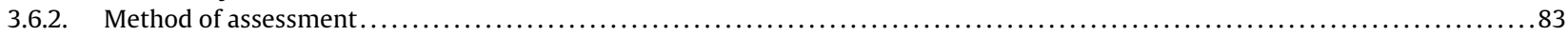

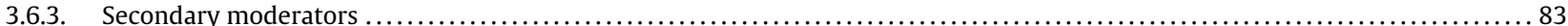

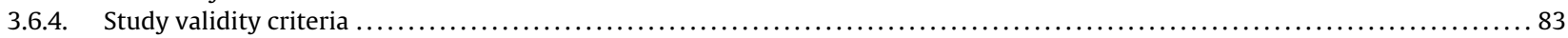

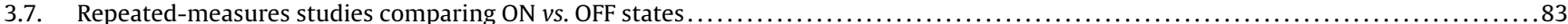

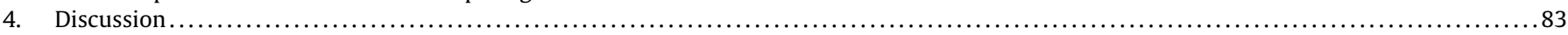

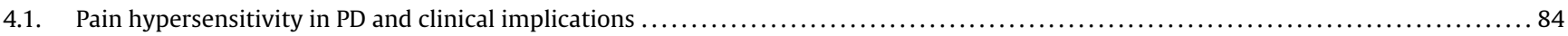

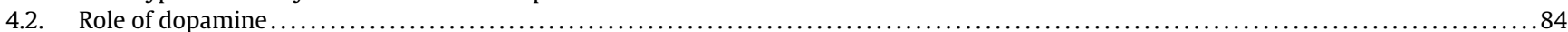

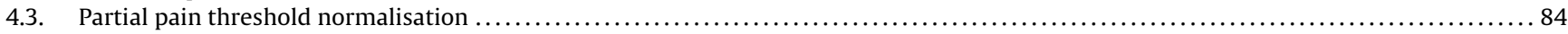

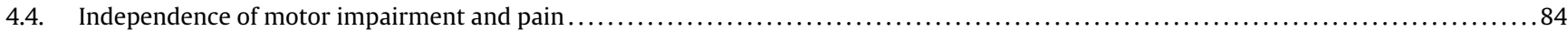

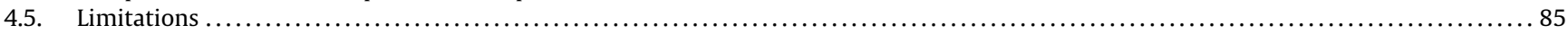

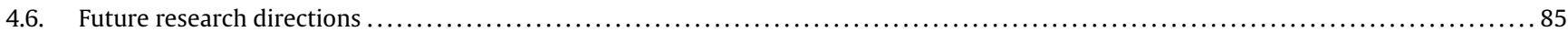

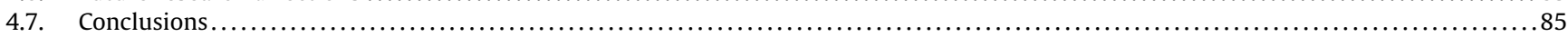

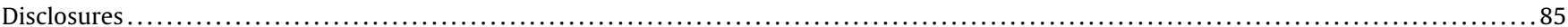

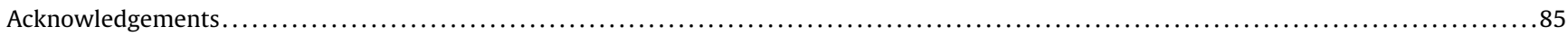

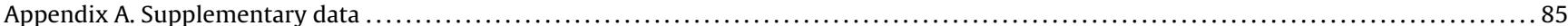

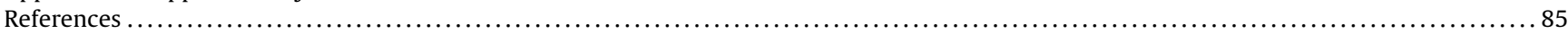

\section{Introduction}

Chronic pain is a common non-motor symptom of Parkinson's disease (PD). A recent systematic review indicated a mean pain prevalence of 68\% in PD patients (Broen et al., 2012), with another study finding that chronic pain complaints, especially musculoskeletal pain, were twice as likely and reported as twice as intense in PD patients compared to age-matched controls with other chronic disorders (Nègre-Pagès et al., 2008). Pain often appears early in the development of PD and may be present years before clinical diagnosis (Schrag et al., 2015). Pain has been rated as the most burdensome non-motor symptom (Chaudhuri and Odin, 2010), and contributes to PD-related disability, sleep disturbance, and impaired quality of life (Chaudhuri and Schapira, 2009; Fil et al., 2013; Quittenbaum and Grahn, 2004). Non-motor symptoms including pain are also a frequent cause of hospitalisation and institutionalisation of PD patients and can increase healthcare costs by up to four times (Chaudhuri and Schapira, 2009). Nevertheless, pain is a frequently overlooked symptom of $\mathrm{PD}$, often unreported by patients unaware that painful symptoms are linked to the disease (Mitra et al., 2008), and consequently under-treated (Broen et al., 2012) which can increase the overall burden of PD. This is especially unfortunate given that pain represents a non-motor symptom that is eminently treatable (Chaudhuri et al., 2010).

While pain in PD is often precipitated by muscular rigidity and/or postural abnormalities (Ford, 2010), neurodegenerative processes could potentially affect not only motor function, but also peripheral (Nolano et al., 2008) and brain (Fil et al., 2013) pathways involved in pain processing. For example, degradation of dopamine-producing cells in the substantia nigra may impair natural analgesia by disrupting the dopamine-mediated descending pathways that block transmission of ascending nociceptive signals from the spinal cord (Fil et al., 2013). A role of dopamine in pain is consistent with reduced pain sensitivity seen in schizophrenia (Stubbs et al., 2015), a disorder linked to dopamine dysregulation, and the possible partial restoration of normal pain thresholds in PD during functional ON states following treatment with dopaminergic agents (Cury et al., 2016).

If pain processing is affected centrally in $\mathrm{PD}$, as hypothesised, this could result in a generalised hypersensitivity to noxious sensations (Cury et al., 2016), which may influence the onset of and/or exacerbate painful symptoms in PD (Broen et al., 2012). Evidence for this hypersensitivity is, however, inconsistent. While several studies have found increased pain sensitivity in PD patients compared to healthy controls (HCs) in response to noxious experimental stimulation (Chen et al., 2015; Lim et al., 2008; Mylius et al., 2009), others have failed to find such an effect (Granovsky et al., 2013; Massetani et al., 1989; Vela et al., 2007). This inconsistency may be influenced by methodological differences across studies, including variation in sample size, dopaminergic and analgesic medications, disease duration and symptom severity (Fil et al., 2013; Priebe et al., 2016). Nevertheless, to our knowledge, there has been no systematic effort to synthesize available evidence from experimental studies and to explore potential sources of study heterogeneity using meta-analytic techniques. Examining the influence of dopamine medication may be especially revealing, both to provide evidence for possible mechanisms of action and for informing potential analgesic treatment.

We therefore conducted a systematic review and meta-analysis of studies comparing PD patients and HCs in their response to noxious experimental stimuli to: (1) examine whether PD patients and HCs differ in their response to experimentally-induced pain; (2) quantify the magnitude of this difference; and (3) explore potential moderators of this association including dopaminergic agents, disease duration, and symptom severity.

\section{Method}

This systematic review and meta-analysis were conducted in accordance with the Preferred Reporting Items for Systematic Reviews and Meta-Analyses (PRISMA) statement (Moher et al., 2009) and the Meta-analysis of Observational Studies in Epidemiology (MOOSE) 
guidelines (Stroup et al., 2000) for observational studies. An a priori established but unpublished protocol was followed.

\subsection{Eligibility criteria}

The following inclusion criteria were applied: (1) use of a group with primary (idiopathic) Parkinson's disease (PD), based on standardized diagnostic criteria (e.g. UK Brain Bank); (2) inclusion of a comparative healthy control (HC) group without PD; (3) application of an experimental pain stimulus; and (4) a quantitative assessment of pain. We excluded studies using participants with secondary Parkinsonism only (e.g. from toxin exposure) and those published in languages other than English.

\subsection{Search strategy}

EMBASE, MEDLINE and PsycINFO databases were independently searched by two reviewers (KG, TT) with the final search performed on 10th October 2016. The following search terms were used: (Parkinson's disease(MeSH) OR Parkinson's) AND (pain(MeSH) OR pain OR nociception) to identify the largest possible pool of potentially eligible studies. The search results were a posteriori refined using limits of 'human studies' and 'English language'. This search strategy was augmented through hand searching reference lists of included articles and relevant reviews.

\subsection{Study selection}

After removal of duplicates, two reviewers (KG, TT) independently screened titles/abstracts for eligibility, and resolved disagreements through consensus. The full-text of potentially eligible articles was then independently scrutinized by two authors (TT, BS). Following consensus, a full-list of eligible articles was defined. When a study provided insufficient data for inclusion, corresponding authors were contacted up to 3 times over an 8-week period to request additional data. Of 8 author groups contacted, 6 (Aschermann et al., 2015; Granovsky et al., 2013; Hara et al., 2013; Nandhagopal et al., 2010; Nolano et al., 2008; Takeda et al., 2013) provided data sufficient to permit study inclusion.

\subsection{Pain outcome variables}

The following pain outcomes were used: (1) pain threshold (the point at which pain is first reported), (2) pain tolerance (the point at which pain is reported as no longer tolerable), and (3) self-report ratings of pain intensity/affect. We used these multiple outcomes to assess whether different aspects of the pain experience were selectively affected in PD. Threshold involves low-intensity pain and is influenced primarily by sensory processes (e.g., localization and initial detection), whereas tolerance is a suprathreshold measure more strongly influenced by affective mechanisms (Apkarian et al., 2005). Pain rating scales provide an easily interpretable index of subjective pain and typically assess sensory (e.g., intensity) or affective (e.g., discomfort) dimensions of pain on a VAS or numerical rating scale.

Sensory threshold (the point at which sensation is first reported) was included as a secondary measure to examine whether PD was also associated with non-painful sensory impairment. As we only wished to examine direct measures of pain, we did not examine supplementary physiological data.

\subsection{Data extraction}

Extraction and coding of study data were performed by two authors (TT, KG), on a standardized form adapted from our previous studies (Stubbs et al., 2015; Thompson et al., 2016). The following data were extracted where available: (1) sociodemographic variables; (2) For PD groups: mean disease duration (years), symptom severity score, functional state (ON/OFF), body side tested (least/most affected), diagnostic criteria used, cognitive impairment, usual treatment, \% of sample with PD-based clinical pain; (3) pain outcomes and pain induction method. Means and standard deviations for each pain outcome were recorded, and any other available information that allowed effect size computation (Lipsey and Wilson, 2001). To reduce reporting bias, authors were contacted for statistical details when findings were simply reported as 'non-significant'.

A number of decisions were made when computing effect sizes from extracted data. First, a few studies $(k=3)$ provided data from multiple independent participant samples (Aschermann et al., 2015; Schestatsky et al., 2007; Vela et al., 2007), e.g. with/without dyskinesia, and were treated as separate studies in the analysis (Borenstein et al., 2009). Second, for one study that used a wide range of temperatures (Nandhagopal et al., 2010), only those eliciting a self-report of pain $\left(47.5^{\circ} \mathrm{C}\right.$ and $\left.49.5^{\circ} \mathrm{C}\right)$ were included. Third, for studies $(k=2)$ that reported the use of different noxious electrical frequencies (Aschermann et al., 2015; Chen et al., 2015), the lowest frequency was arbitrarily selected, as there appears to be no persuasive evidence for a frequency effect (Chen et al., 2015). Fourth, where studies performed repeated pain assessments on the same set of participants (e.g. across different stimuli), multiple effect sizes were computed for each assessment with any dependency across effect sizes modeled using robust variance estimation (RVE) (see Section 2.7.2).

\subsection{Study validity criteria}

Two authors (KG, TT) independently rated each study on several dichotomous validity criteria, with a third author (BS) available for mediation in the event of disagreement. We assessed case/control comparability and participant selection with 5 items from the Newcastle Ottawa Scale (Wells et al., 2008), and methodological soundness with 14 items based on Cochrane Collaboration principles as reviewed by Deeks et al. (2003) (see Table 1). Overall scores were not computed due to concerns over their interpretability (Deeks et al., 2003), but the impact of poorly endorsed validity criteria was examined in moderator analysis.

\subsection{Statistical analysis}

\subsubsection{Effect size}

A standardized mean difference (SMD) for PD vs. control groups was computed for each study using Hedges' $g$ formula (Borenstein et al., 2009). Hedges' $g$ is equivalent to Cohen's $d$, but corrects for bias in small samples. Effect sizes can be interpreted as $0.20,0.50$ and 0.80 corresponding to small, medium and large effects respectively (Cohen, 1988).

Effect size (ES) was coded so that positive values indicated higher pain in the PD compared to the HC group.

\subsubsection{Meta-analysis}

An overall summary effect size for each outcome was estimated using random-effects meta-analysis. A random-effects model was chosen as heterogeneity was expected based on similar metaanalyses conducted for other disease conditions (Stubbs et al., 2015; Thompson et al., 2016). Several studies reported multiple ES data from the same samples and a few studies used different PD sample but with the same control group comparisons. Conventional meta-analytic techniques that assume independence of effect sizes were therefore not considered appropriate. Instead, we used a robust variance estimation (RVE) method (Hedges et al., 2010), 
Table 1

Summary of included studies.

\begin{tabular}{|c|c|c|c|c|c|c|c|c|c|}
\hline Study & N-PD & $\begin{array}{l}\text { Duration } \\
\text { years }\end{array}$ & UPDRS-III & $\begin{array}{l}\text { ON/OFF state } \\
\text { during pain } \\
\text { testing }\end{array}$ & Usual Medication & $\mathrm{N}-\mathrm{CON}$ & Modality & Testing Site & Pain Measure \\
\hline Priebe et al. (2016) & 23 & 8.1 & 18.4 (OFF) & $\begin{array}{l}\text { OFF } \\
\text { ON }\end{array}$ & $\begin{array}{l}\text { Mixed }(\mathrm{L}-\mathrm{DOPA}=1 ; \text { DA } \\
\text { agonists = 5; } \\
\text { L-DOPA+DA } \\
\text { agonists = 17; MAO } \\
\text { inhibitors = 9; COMT } \\
\text { inhibitors = 2; NMDA } \\
\text { blockers =5) }\end{array}$ & 23 & $\begin{array}{l}\text { Heat } \\
\text { Electrical }\end{array}$ & $\begin{array}{l}\text { Forearm } \\
\text { Leg }\end{array}$ & $\begin{array}{l}\text { Pain } \\
\text { Threshold } \\
\text { Pain } \\
\text { Threshold } \\
\text { (NFR) } \\
\text { Pain } \\
\text { Intensity } \\
\text { EMG } \\
\text { response }\end{array}$ \\
\hline Mylius et al. (2016) & 14 & 1.8 & $22.8(\mathrm{ON})$ & OFF & NS but inc. L-DOPA & 27 & $\begin{array}{l}\text { Heat } \\
\text { Electrical }\end{array}$ & $\begin{array}{l}\text { Forearm } \\
\text { Leg }\end{array}$ & $\begin{array}{l}\text { Pain } \\
\text { Threshold } \\
\text { Pain } \\
\text { Threshold } \\
\text { (NFR) }\end{array}$ \\
\hline Allen et al. (2016) & 26 & - & $27.3^{\mathrm{b}}(\mathrm{ON})$ & ON & NS & 11 & $\begin{array}{l}\text { Pressure (type } \\
\text { not stated) }\end{array}$ & Upper Arm Leg & Pain Threshold \\
\hline $\begin{array}{l}\text { Aschermann et al. } \\
\text { (2015) }\end{array}$ & 6 & 6.20 & $11.2(\mathrm{ON})$ & ON & $\begin{array}{l}\text { Mixed (levodopa, } \\
\text { dopamine agonists, } \\
\text { MAO and } \\
\text { COMT-inhibitors) }\end{array}$ & 6 & Heat & Forearm & $\begin{array}{l}\text { Pain Threshold (for } \\
\text { moderate pain) } \\
\text { Pain Intensity }\end{array}$ \\
\hline $\begin{array}{l}\text { Aschermann et al. } \\
\text { (2015) }\end{array}$ & 6 & 5.50 & $15.8(\mathrm{ON})$ & ON & Mixed (as above) & $6^{a}$ & Heat & Forearm & $\begin{array}{l}\text { Pain Threshold (for } \\
\text { moderate pain) } \\
\text { Pain Intensity }\end{array}$ \\
\hline Chen et al. (2015) & 72 & 4.90 & $\begin{array}{l}29.5 \text { (OFF) } \\
23.1(\mathrm{ON})\end{array}$ & $\begin{array}{l}\text { OFF } \\
\text { ON }\end{array}$ & L-DOPA & 35 & Electrical & Hand & $\begin{array}{l}\text { Sensory } \\
\text { Threshold } \\
\text { Pain } \\
\text { Tolerance }\end{array}$ \\
\hline Grashorn et al. (2015) & 25 & 3.70 & $\begin{array}{l}24.1 \text { (OFF) } \\
20.7(\mathrm{ON})\end{array}$ & $\begin{array}{l}\text { OFF } \\
\text { ON }\end{array}$ & $\begin{array}{l}\text { Mixed }(\mathrm{L}-\mathrm{DOPA}=4, \\
\text { L-DOPA+ MAO } \\
\text { inhibitors = 2, L-DOPA+ } \\
\text { DA agonists = 1; DA } \\
\text { agonists = 7; DA } \\
\text { agonists + MAO } \\
\text { Inhibitors =9; MAO } \\
\text { inhibitors = 2) }\end{array}$ & 30 & $\begin{array}{l}\text { Heat } \\
\text { Cold } \\
\text { pressor }\end{array}$ & $\begin{array}{l}\text { Forearm } \\
\text { Leg }\end{array}$ & $\begin{array}{l}\text { Pain Threshold (for } \\
\text { moderate pain) } \\
\text { Pain Intensity }\end{array}$ \\
\hline Tan et al. (2015) & 14 & 2.50 & 21.8 (OFF) & OFF & None & 17 & Heat & Forearm & $\begin{array}{l}\text { Sensory Threshold Intensity } \\
\text { Pain Threshold } \\
\text { Pain }\end{array}$ \\
\hline Takeda et al. (2014) & 23 & 5.60 & $27.0(\mathrm{ON})$ & ON & $\begin{array}{l}\text { Mixed (L-DOPA = 4, } \\
\text { L-DOPA+ DA } \\
\text { agonists }=11 \text {, MAO } \\
\text { inhibitors }=8 \text { ) }\end{array}$ & 12 & Electrical & $\begin{array}{l}\text { Hand } \\
\text { Leg }\end{array}$ & $\begin{array}{l}\text { Pain Threshold } \\
\text { (for moderate } \\
\text { pain) }\end{array}$ \\
\hline
\end{tabular}


Table 1 (Continued)

\begin{tabular}{|c|c|c|c|c|c|c|c|c|c|}
\hline Study & $\mathrm{N}-\mathrm{PD}$ & $\begin{array}{l}\text { Duration } \\
\text { years }\end{array}$ & UPDRS-III & $\begin{array}{l}\text { ON/OFF state } \\
\text { during pain } \\
\text { testing }\end{array}$ & Usual Medication & $\mathrm{N}-\mathrm{CON}$ & Modality & Testing Site & Pain Measure \\
\hline Hara et al. (2013) & 42 & 6.50 & $21.6(\mathrm{ON})$ & ON & $\begin{array}{l}\text { Mixed (L-DOPA, DA } \\
\text { agonists, MAO } \\
\text { inhibitors, } \\
\text { catechol-Omethyl } \\
\text { transferase and } \\
\text { amantadine) }\end{array}$ & 17 & Electrical & Face & Pain Threshold \\
\hline Granovsky et al. (2013) & 23 & 6.30 & $23.6(\mathrm{ON})$ & $\begin{array}{l}\text { OFF } \\
\text { ON }\end{array}$ & $\begin{array}{l}\text { Mixed (L-DOPA =11, } \\
\text { DA agonists }=17, \text { MAO } \\
\text { inhibitors }=19 \text {, } \\
\text { Anticholinergics }=8 \text {, } \\
\text { Amantadine }=14 \text { ) }\end{array}$ & 19 & $\begin{array}{l}\text { Heat } \\
\text { Pressure } \\
\text { (von Frey } \\
\text { filaments) }\end{array}$ & $\begin{array}{l}\text { Hand } \\
\text { Forearm }\end{array}$ & $\begin{array}{l}\text { Pain } \\
\text { Threshold } \\
\text { Pain } \\
\text { Intensity }\end{array}$ \\
\hline Vela et al. (2012) & 18 & 11.60 & $\begin{array}{l}34.5(\mathrm{OFF}) \\
22.1(\mathrm{ON})\end{array}$ & $\begin{array}{l}\text { OFF } \\
\text { ON }\end{array}$ & $\begin{array}{l}\text { Mixed (L-DOPA, DA } \\
\text { agonists) }\end{array}$ & 18 & $\begin{array}{l}\text { Pressure } \\
\text { (algometer) } \\
\text { Heat } \\
\text { Cold }\end{array}$ & $\begin{array}{l}\text { Neck } \\
\text { Head } \\
\text { Hand } \\
\text { Leg }\end{array}$ & Pain Threshold \\
\hline $\begin{array}{l}\text { Ciampi de Andrade } \\
\text { et al. (2012) }\end{array}$ & 25 & 15.10 & $\begin{array}{l}42.7(\mathrm{OFF}) \\
25.8(\mathrm{ON}- \\
\text { via } \mathrm{DBS})\end{array}$ & $\begin{array}{l}\text { OFF } \\
\text { ON }\end{array}$ & L-DOPA & 35 & $\begin{array}{l}\text { Heat } \\
\text { Cold } \\
\text { Pressure } \\
\text { (von Frey } \\
\text { filaments) }\end{array}$ & Hand & $\begin{array}{l}\text { Sensory } \\
\text { Threshold } \\
\text { Pain Threshold } \\
\text { Pain Intensity }\end{array}$ \\
\hline Stamelou et al. (2012) & 19 & 6.70 & $22.4(\mathrm{ON})$ & OFF & $\begin{array}{l}\text { L-DOPA (no details on } \\
\text { other medications) }\end{array}$ & 17 & $\begin{array}{l}\text { Heat } \\
\text { Electrical }\end{array}$ & $\begin{array}{l}\text { Forearm } \\
\text { Leg }\end{array}$ & $\begin{array}{l}\text { Pain Threshold } \\
\text { (NFR) } \\
\text { Pain Threshold }\end{array}$ \\
\hline Mylius et al. (2011) & 29 & 7.40 & $25.6(\mathrm{ON})$ & OFF & $\begin{array}{l}\text { L-DOPA (no details on } \\
\text { other medications) }\end{array}$ & 27 & $\begin{array}{l}\text { Electrical } \\
\text { Heat }\end{array}$ & $\begin{array}{l}\text { Forearm } \\
\text { Leg }\end{array}$ & $\begin{array}{l}\text { Pain Threshold } \\
\text { (NFR) }\end{array}$ \\
\hline Maruo et al. (2011) & 17 & 15.50 & $36.3(\mathrm{ON})$ & ON & $\begin{array}{l}\text { L-DOPA, DA agonists } \\
\text { (no details on other } \\
\text { medications) }\end{array}$ & 14 & $\begin{array}{l}\text { Cold } \\
\text { Heat }\end{array}$ & Hand & $\begin{array}{l}\text { Sensory } \\
\text { Threshold } \\
\text { Pain Threshold }\end{array}$ \\
\hline Zambito et al. (2011) & 106 & 5.70 & 23.5 (OFF) & OFF & $\begin{array}{l}\text { Mixed }(\text { L-DOPA }=38 \\
\text { DA agonists = } 19 ; \\
\text { L-DOPA + DA } \\
\text { agonists }=49)\end{array}$ & 51 & Electrical & $\begin{array}{l}\text { Hand } \\
\text { Foot }\end{array}$ & $\begin{array}{l}\text { Sensory } \\
\text { Threshold } \\
\text { Pain Threshold } \\
\text { Pain Tolerance }\end{array}$ \\
\hline
\end{tabular}




\begin{tabular}{|c|c|c|c|c|c|c|c|c|c|}
\hline $\begin{array}{l}\text { Nandhagopal et al. } \\
\text { (2010) }\end{array}$ & 12 & 9.40 & $\begin{array}{l}28.8(\mathrm{OFF}) \\
15.0(\mathrm{ON})\end{array}$ & $\begin{array}{l}\text { OFF } \\
\text { ON }\end{array}$ & $\begin{array}{l}\text { Mixed (L-DOPA } \\
\text { with/without } \\
\text { other medica- } \\
\text { tion =12) }\end{array}$ & 13 & Heat & Forearm & $\begin{array}{l}\text { Pain Intensity } \\
\text { Pain } \\
\text { Unpleasantness }\end{array}$ \\
\hline Mylius et al. (2009) & 15 & 11.00 & 28.3 (OFF) & OFF & Mixed & 18 & $\begin{array}{l}\text { Heat } \\
\text { Electrical }\end{array}$ & $\begin{array}{l}\text { Forearm } \\
\text { Leg }\end{array}$ & $\begin{array}{l}\text { Pain Threshold } \\
\text { Pain Threshold } \\
\text { (NFR) }\end{array}$ \\
\hline Nolano et al. (2008) & 18 & 7.60 & $26.6(\mathrm{ON})$ & ON & $\begin{array}{l}\text { Mixed (L-DOPA } \\
\text { with/without } \\
\text { other medica- } \\
\text { tion }=14 \text {, } \\
\text { None }=4 \text { ) }\end{array}$ & 54 & $\begin{array}{l}\text { Cold } \\
\text { Heat } \\
\text { Pressure (nylon } \\
\text { monofilament) }\end{array}$ & $\begin{array}{l}\text { Hand } \\
\text { Foot }\end{array}$ & $\begin{array}{l}\text { Sensory Threshold } \\
\text { Pain Threshold }\end{array}$ \\
\hline Lim et al. (2008) & 50 & 4.38 & $\begin{array}{l}29.6 \text { (OFF) } \\
21.3(\mathrm{ON})\end{array}$ & $\begin{array}{l}\text { OFF } \\
\text { ON }\end{array}$ & $\begin{array}{l}\text { L-DOPA (no } \\
\text { details on other } \\
\text { medications) }\end{array}$ & 20 & Cold pressor & Hand & $\begin{array}{l}\text { Pain Threshold } \\
\text { Pain Tolerance }\end{array}$ \\
\hline $\begin{array}{l}\text { Gerdelat-Mas et al. } \\
\text { (2007) }\end{array}$ & 13 & 7.30 & $\begin{array}{l}21.2(\mathrm{OFF}) \\
10.8(\mathrm{ON})\end{array}$ & $\begin{array}{l}\text { OFF } \\
\text { ON }\end{array}$ & $\begin{array}{l}\text { Mixed (L-DOPA } \\
\text { and/or DA } \\
\text { agonists) }\end{array}$ & 10 & Electrical & Leg & $\begin{array}{l}\text { Pain Threshold } \\
\text { (NFR) }\end{array}$ \\
\hline $\begin{array}{l}\text { Schestatsky et al. } \\
\text { (2007) }\end{array}$ & 9 & 5.40 & $17.1(\mathrm{NS})$ & $\begin{array}{l}\text { OFF } \\
\text { ON }\end{array}$ & $\begin{array}{l}\text { NS but inc. } \\
\text { L-DOPA }\end{array}$ & 9 & $\begin{array}{l}\text { Heat } \\
\text { Laser }\end{array}$ & Hand & $\begin{array}{l}\text { Sensory Threshold } \\
\text { Pain Threshold }\end{array}$ \\
\hline $\begin{array}{l}\text { Schestatsky et al. } \\
\text { (2007) }\end{array}$ & 9 & 6.00 & $19.1(\mathrm{NS})$ & $\begin{array}{l}\text { OFF } \\
\text { ON }\end{array}$ & $\begin{array}{l}\text { NS but inc. } \\
\text { L-DOPA }\end{array}$ & $9^{\mathrm{a}}$ & $\begin{array}{l}\text { Heat } \\
\text { Laser }\end{array}$ & Hand & $\begin{array}{l}\text { Sensory Threshold } \\
\text { Pain Threshold }\end{array}$ \\
\hline Vela et al. (2007) & 25 & 11.38 & $24.6(\mathrm{ON})$ & ON & NS & 25 & $\begin{array}{l}\text { Pressure } \\
\text { (algometer) }\end{array}$ & Hand & Pain Threshold \\
\hline Vela et al. (2007) & 25 & 4.72 & $19.9(\mathrm{ON})$ & ON & NS & $25^{\mathrm{a}}$ & $\begin{array}{l}\text { Pressure } \\
\text { (algometer) }\end{array}$ & Hand & Pain Threshold \\
\hline $\begin{array}{l}\text { Brefel-Courbon et al. } \\
\text { (2005) }\end{array}$ & 9 & 9.60 & $\begin{array}{l}25.0 \text { (OFF) } \\
15.0(\mathrm{ON})\end{array}$ & $\begin{array}{l}\text { OFF } \\
\text { ON }\end{array}$ & $\begin{array}{l}\text { Mixed (L-DOPA } \\
\text { and/or DA } \\
\text { agonists) }\end{array}$ & 9 & Cold pressor & Hand & Pain Threshold \\
\hline Djaldetti et al. (2004) & 51 & 5.30 & 24.0 (OFF) & OFF & $\begin{array}{l}\text { Mixed (L-DOPA } \\
\text { and/or DA } \\
\text { agonists) }\end{array}$ & 28 & Heat & Hand & Pain Threshold \\
\hline Massetani et al. (1989) & 15 & 4.10 & NA & ON & $\begin{array}{l}\text { Mixed (L-DOPA } \\
\text { with or } \\
\text { without } \\
\text { peripheral } \\
\text { decarboxylase } \\
\text { inhibitor }=10 \text {, } \\
\text { drug free }=5 \text { ) }\end{array}$ & 8 & Electrical & Face & $\begin{array}{l}\text { Sensory Threshold } \\
\text { Pain Threshold } \\
\text { Pain Threshold } \\
\text { (blink reflex) }\end{array}$ \\
\hline $\begin{array}{l}\text { TOTAL } \\
\text { for } 26 \text { studies }\end{array}$ & 739 & $M=7.10$ years & $\begin{array}{l}\text { ON: } \\
M=22.2 \mathrm{yrs} \\
\text { OFF: } \\
M=27.0 \mathrm{yrs}\end{array}$ & $\begin{array}{l}\mathrm{ON}=19 ; \\
\mathrm{OFF}=18\end{array}$ & $\begin{array}{l}\text { L-DOPA } \\
\text { with/without } \\
\text { others }=23 \\
\text { Not Stated }=2 \\
\text { None }=1\end{array}$ & 553 & $\begin{array}{l}\text { Heat }=16 ; \\
\text { Electrical =1; } \\
\text { Cold = 7; } \\
\text { Pressure =6; } \\
\text { Laser }=1\end{array}$ & $\begin{array}{l}\text { Hand }=13 ; \\
\text { Forearm }=10 ; \\
\text { Leg }=10 ; \text { Face }=2 ; \\
\text { Foot }=2 ; \\
\text { Neck/Head } / \text { Upper } \\
\text { Arm = } 1\end{array}$ & $\begin{array}{l}\text { Pain } \\
\text { Threshold =21; } \\
\text { Sensory } \\
\text { Threshold =9; } \\
\text { Intensity =7; } \\
\text { Suprathreshold } \\
\text { (moderate } \\
\text { pain/tolerance) =6; } \\
\text { Unpleasantness = }\end{array}$ \\
\hline
\end{tabular}

Key: NS = Not Stated, NFR = Nociceptive Flexion Reflex, DA = Dopamine, MAO = Monoamine oxidase, COMT = Catechol-O-methyltransferase, NMDA = N-methyl-D-aspartate

a Same control group used for '-a' and '-b' studies.

b Estimated from MDS-UPDRS-III score of 33.8 using conversion formula (Goetz et al., 2012). 
which adjusts individual ES weights based on the degree of their dependency. We employed a relatively new version of RVE for correlated effect sizes, which provides reliable estimates even when relatively low numbers of studies are available. Simulation studies have demonstrated accurate estimation, provided that the adjusted RVE degrees of freedom (df) $>4$ (Tipton, 2015), and this criterion is used here ( $d f$ is larger when study number is high and when multiple effect sizes from a study are relatively independent). RVE estimates dependency from the data itself, and therefore is a superior approach to averaging across conditions, which results in both information loss and relies on a knowledge of the correlations of outcomes across conditions which are rarely reported (Fisher and Tipton, 2014).

Separate analyses were conducted for OFF/ON functional states, to examine whether pain sensitivity is altered by treatment (typically dopaminergic medication). For the specific outcome of pain ratings, we only examined $k=5$ out of 7 studies where stimulation intensity was identical for both the PD and control groups (i.e., where a fixed-intensity/fixed-time paradigm was used), to avoid any confounding of group differences in pain ratings with group differences in stimulation intensity.

\subsubsection{Meta-regression analyses}

If effect sizes showed moderate or greater inconsistency across studies as assessed by Higgin's $\mathrm{I}^{2}$ (Higgins et al., 2003), with values of $25 \%, 50 \%$ and $75 \%$ corresponding to low, moderate and high inconsistency, meta-regression was conducted to identify possible underlying sources of variation.

First, we examined symptom severity (as measured by the UPDRS-III), disease duration (years) and, for pain threshold, the assessment method (limits vs. constant/adjusted stimuli) as primary moderators, based on a rationale defined a priori. Greater symptom severity and longer disease duration likely to reflect increased neurodegeneration and therefore may be associated with a greater degree of abnormal pain perception. In addition, the methods of limits (where intensity increases to a pre-defined limit) involves a reaction time artefact (e.g. from pressing a button) which could artificially inflate threshold times and underestimate true levels of pain selectively for PD patients due to motor impairment (Cury et al., 2016). Conversely, the methods of constant stimuli/adjustment (where constant temperatures are gradually adjusted) (see Yarnitsky and Pud, 2004) contains no reaction time component.

Secondary moderators were study gender composition, age, stimulus modality, PD side subject to pain testing (least/most affected), anatomic site subject to pain testing, and sample percentage experiencing PD-based pain complaints. These variables were examined to provide preliminary data on any potential moderating influence, as all have been suggested as possible influences (Fil et al., 2013). Additionally, meaningful study validity criteria were assessed as moderators, such as case and control selection, criteria-based PD diagnosis, explicit mentioning of disallowing pain medications. Finally, where the endorsement of important validity criteria varied across studies, the influence of these criteria as potential moderators of effect size was also assessed.

\subsection{Publication bias}

Publication bias was examined through visual inspection of funnel plots of mean study ESs against standard errors. Any visual asymmetry resulting from the absence of small sample studies with small ESs can suggest possible publication bias. Asymmetry was also tested statistically with Egger's bias test (Egger et al., 1997) with $p<0.05$ indicating asymmetry. If present, a revised effect size assuming the presence of publication bias was computed using the trim and fill method (Duval and Tweedie, 2000).
As the citation refers to a package (robumeta) within a computer program (R): all analyses were performed using the robumeta package (Fisher and Tipton, 2014) in R (R Core Team, 2014).

\section{Results}

\subsection{Study selection}

3047 unique hits were identified through database searches, with 6 additional records identified through manual searching of reference lists. Following the initial screening of abstracts, 47 articles were retained for full text review of which 21 were excluded, resulting in a total of 26 studies retained for analysis (Fig. 1).

\subsection{Participant characteristics}

The 26 retained studies provided aggregated data for $\mathrm{N}=1292$ participants, consisting of 739 patients and 553 HCs. The mean study age ( $k=26$ studies) was 63.8 years $(S D=3.0$, range of means $=58.8-69.9$ years) for the aggregated PD sample, and 62.4 years $(S D=3.9$, range of means $=54.8-71.4$ years $)$ for the aggregated HC sample. The PD sample consisted of $35.7 \%$ females and the HC sample consisted of $42.7 \%$ of females $(k=26)$.

For the PD sample, mean disease duration $(k=25)$ was 7.1 years $(S D=3.4$, range of means $=1.8-15.5)$. Symptom severity $(k=25)$ was most commonly measured with the original UPDRS-III Motor Examination scale (Fahn and Elton, 1987) and was assessed during both ON ( $k=19$; UPDRS-III $M=21.8)$ and OFF ( $k=13$; UPDRS-III $M=27.0)$ functional states. ON states were achieved with antiParkinson medication $(k=18)$ or deep brain stimulation $(k=1)$.

A diagnosis of PD was based on UKBBC $(k=18)$, ICD-10:G20 $(k=1)$, Gelb NINDS guidelines $(k=1)$ or was simply reported as a clinical diagnosis of PD $(k=6)$. Medication data $(k=24)$ included a single study which used a medication-naïve patient sample (Tan et al., 2015), while 23 studies reported regular usage of antiParkinson medication (L-DOPA with/without other medication) by some or all enrolled patients $(M=95 \%$, study range $=67-100 \%)$. Most studies $(k=22)$ specified a minimum level of cognitive functioning for inclusion in the study, with scores $\geq 25$ on the Mini-Mental State Examination being the most extensively used inclusion criterion $(k=15)$.

Presence or absence of a primary chronic pain condition was reported by 13 studies. Of these, 12 reported no co-occurring chronic pain conditions and a single study reported that 2 patients may have exhibited chronic low back pain. The mean study percentage of $\mathrm{PD}$ patients reporting secondary pain complaints attributable to $\operatorname{PD}(k=18)$ was $48 \%$.

\subsection{Study characteristics}

Of the 26 studies, 15 were conducted in Europe, 6 in Asia, 2 in Oceania and 1 in each of North America, South America and Africa. All studies reported the method of pain induction and functional state during testing. Pain induction methods comprised heat $(k=16)$, electrical $(k=10)$, cold $(k=7)$, pressure $(k=6)$ and laser $(k=1)$. Location of pain induction $(k=24)$ for patients was on the most affected body side $(k=9)$, least affected side $(k=3)$ or the left or right side $(k=12)$, and was applied to the hand $(k=13)$, forearm $(k=10)$, leg $(k=10)$, face $(k=2)$, neck $(k=1)$, head $(k=1)$ or upper $\operatorname{arm}(k=1)$. Pain testing was performed during the ON state $(k=19)$, the OFF state $(k=18)$, with 11 studies having assessed pain during both states in a repeated-measures design. For ON states, pain testing was usually conducted within the first hour of treatment initiation, with the presence of $\mathrm{ON}$ states confirmed by a reduction in UPDRS-III motor scores in all 7 repeated-measures design studies that assessed this. 


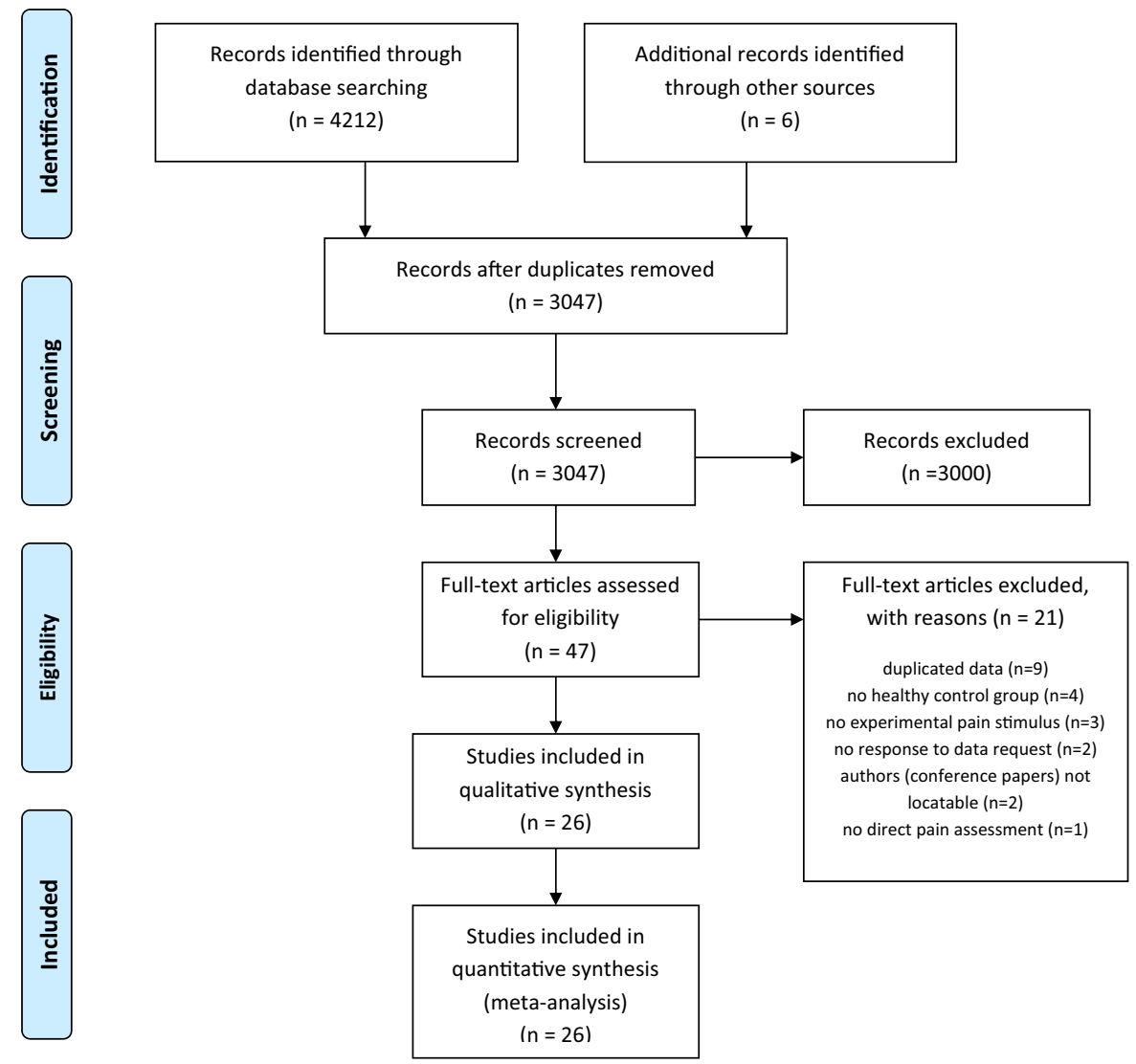

Fig. 1. PRISMA flow diagram.

The majority of studies used pain threshold $(k=21)$ as the method of pain assessment ( $k=11$ method of limits, $k=7$ method of levels/adjustment, $k=3$ not stated), with other outcomes including pain intensity ratings $(k=7)$, pain tolerance $(k=3)$ and threshold for moderate pain $(k=3)$, with several individual studies employing multiple induction and assessment methods. Nine studies also assessed sensory threshold. Aside from one study that included only drug-naïve patients (Tan et al., 2015), OFF states were achieved by the withdrawal of dopaminergic medication, with pain testing usually taking place after $\mathrm{a} \geq 12$-h washout period.

A summary of the key characteristics of the 26 individual studies is presented in Table 1 .

\subsection{Study validity criteria}

Acceptable agreement across the two raters was found for most items (Kappa $=0.77-1.00)$ except for those with high rates of endorsement ( $\min$ Kappa=0.25). High endorsement rates can result in low kappa values due to marginal homogeneity (Gwet, 2002) and thus percentage agreement across raters was computed, with resultant percentages indicating good agreement $(>87 \%)$ for those items. Complete consensus was reached whenever any disagreement occurred (see Appendix S1 for all item ratings).

Ratings indicated methodological soundness (e.g., reporting of functional state during testing, complete data provided, clear description of procedures) for the majority of studies ( $>85 \%$ for most criteria). Most studies excluded patients with comorbid depression (77\%) or somatosensory disorder (73\%), with $42 \%$ explicitly specifying that use of pain medication $(<24 \mathrm{~h})$ was an exclusion criterion. Low endorsement of 'selection of PD cases (23\%) and controls (27\%) validity criterion reflected limited detail on recruitment methods; although studies did generally state the name of the recruiting hospital and provide good descriptions of characteristics of controls, usually matching cases and controls either experimentally or by statistically controlling for age/gender (77\%).

Some statistical inconsistencies were noted. One study (Nolano et al., 2008) reported implausible pain thresholds $\left(9.1-12.4^{\circ} \mathrm{C}\right)$ for heat stimulation (values for other stimuli were plausible), and a further study (Schestatsky et al., 2007) reported means and SDs for pain threshold highly inconsistent with reported $p$-values (even when $S D$ s were treated as $S E M$ s), and so this specific data were excluded.

\subsection{Meta-analysis results}

\subsubsection{Pain threshold}

Pain threshold data was examined for $k=20$ studies comprising a total sample of $\mathrm{N}=926(\mathrm{n}=577 \mathrm{PD}, \mathrm{n}=473 \mathrm{HCs})$, with PD patents assessed during both OFF $(k=14 ; \mathrm{N}=728)$ and/or ON $(k=13 ; \mathrm{N}=617)$ states. Seven repeated-measures studies provided pain threshold data for both ON and OFF states.

Meta-analysis of pain threshold, aggregating data from both ON and OFF states, found significantly lower overall pain threshold (i.e., greater pain sensitivity) in PD patients compared to HCs $\left(S M D=0.37, \mathrm{Cl}_{95}[0.16,0.57], p=0.001\right)$. Moderate to high heterogeneity $\left(\mathrm{I}^{2}=63 \%\right)$ was observed and $k=16$ of 20 studies reported lower pain thresholds in PD.

When separate meta-analyses were performed for ON and OFF state data, significantly lower pain thresholds in PD patients compared to HCs were found for the OFF state $(k=14 ; S M D=0.51$, $\left.\mathrm{Cl}_{95}[0.23,0.79], p=0.002\right)$, along with moderate-high heterogeneity $\left(\mathrm{I}^{2}=64 \%\right)$. For ON state data, lower overall pain threshold in PD 


\section{Forest Plot}

Studies

Gerdelat-Mas et al (2007)

Mylius et al (2009)

Electric (Nociceptive Flexion Response)

Electric

Heat

Brefel-Courbon et al (2005)

Vela et al (2012)

Pressure - most affected side

Heat - most affected side

Cold - most affected side

Pressure - least affected side

Heat - least affected side

Cold - least affected side

Stamelou et al (2012)

Electric (Nociceptive Flexion Response)

Electric

Heat

Ciampi de Andrade et al (2012)

Pressure

Heat

Cold

Mylius et al (2011)

Electric (Nociceptive Flexion Response)

Electric

Heat

Djaldetti et al (2004)

Zambito-Marsala et al (2011)

Hand

Foot

Lim et al (2008)

Mylius et al (2016)

Heat

Electric

Electric (Nociceptive Flexion Response)

Priebe et al (2016)

Heat

Electric (Nociceptive Flexion Response)

Granovsky et al (2013)

Heat - most affected side

Pressure - most affected side

Heat - least affected side

Pressure - least affected side

Tan et al (2015)
Effect Size Weight

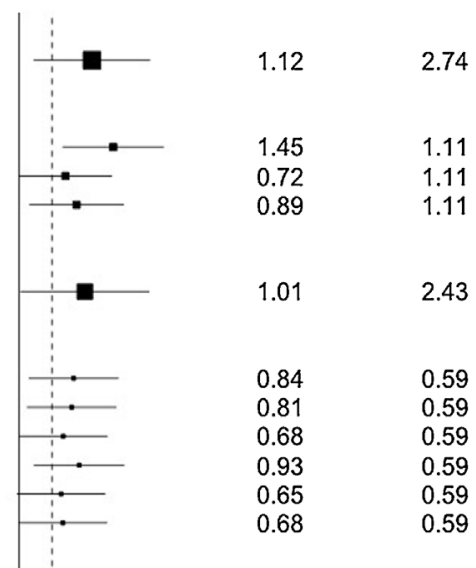

1.18

1.18

1.18

1.42

1.42

1.42

1.40

1.40

1.40

4.56

2.61

2.61

$0.51 \quad 4.29$

$\begin{array}{ll}-0.08 & 1.22\end{array}$

$0.57 \quad 1.22$

$\begin{array}{ll}0.89 & 1.22\end{array}$

$0.30 \quad 2.01$

$0.11 \quad 2.01$

$\begin{array}{ll}-0.59 & 0.96\end{array}$

$\begin{array}{ll}-0.03 & 0.96\end{array}$

$-0.52 \quad 0.96$

$\begin{array}{ll}-0.35 & 0.96\end{array}$

\begin{tabular}{ll}
$-0.81 \quad 3.31$ \\
\hline
\end{tabular}

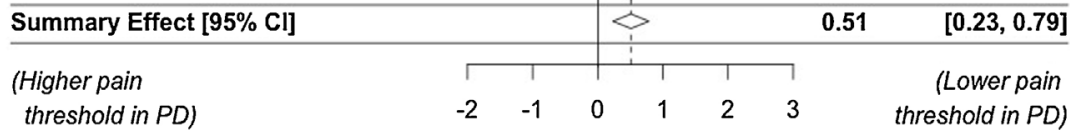

Effect Size

Fig. 2. Forest plot of pain threshold, with box sizes proportional to study weights (for studies with multiple outcomes, weights are divided evenly across outcomes).

was also found but with a reduced effect size $(k=13 ; S M D=0.23$, $\left.\mathrm{Cl}_{95}[0.02,0.45], p=0.04\right)$ and moderate heterogeneity $\left(\mathrm{I}^{2}=49 \%\right)$. A forest plot of pain threshold data for the OFF state is provided in Fig. 2.
Funnel plots of pain threshold data revealed no obvious asymmetry for data from either ON or OFF states, corroborated by non-significant values for Egger's asymmetry test (OFF: $z=0.30$, $\mathrm{p}=0.76 ; \mathrm{ON}: \mathrm{z}=0.42, \mathrm{p}=0.67)$, consistent with a possible lack of publication bias. 


\subsubsection{Suprathreshold pain response}

Three studies reported pain tolerance data, and 3 other studies reported 'threshold to moderate pain' (where participants were told to withdraw from noxious stimulation when experiencing moderate pain). Both measures were collapsed to a single suprathreshold pain category to maximise power (three studies is insufficient for RVE analysis). The 6 combined studies provided aggregate data for 442 participants $(n=288$ PD patients, $n=154$ HCs). Pain assessment occurred during both ON $(k=5)$ and OFF states $(\mathrm{k}=4)$, with 3 studies providing data for both states.

Meta-analysis of overall suprathreshold data found lower tolerance to suprathreshold pain (suggesting greater sensitivity) in PD patients, but this just failed to achieve statistical significance $\left(S M D=0.30, \mathrm{Cl}_{95}[-0.01,0.61], \mathrm{p}=0.06\right)$. Analysis of different functional states indicated lower tolerance to suprathreshold pain during OFF $(k=4 ; S M D=0.44, p=0.04)$ states but not during ON $(k=5 ; S M D=0.15, p=0.28)$ states. For analysis of OFF state data, population confidence intervals (and thus $p$-values) may be wider or narrower (Tipton, 2015) as the adjusted $d f<4$. Low heterogeneity was observed in all instances $\left(\mathrm{I}^{2}=4-27 \%\right)$.

A funnel plot of overall suprathreshold data suggested asymmetry due to the presence of a small sample study with a large negative effect size. Some indication of possible asymmetry emerged from Egger's test, which failed to achieve significance (but is also likely to be underpowered with only 6 studies), $z=1.90, p=0.054$, A revised estimate using trim and fill methods suggested a slightly larger effect size $(S M D=0.36, p=0.01)$ if publication bias is assumed.

\subsubsection{Other outcomes: pain ratings and sensory threshold}

The adjusted $d f$ from RVE meta-analysis of pain ratings and sensory threshold were $<4$ (reflecting both limited number of studies using these assessment methods and data dependency), which precluded reliable estimation of ESs (Tipton, 2015) for these outcomes.

\subsection{Meta-regression analyses: pain threshold}

Meta-regression analyses were conducted to identify underlying sources of heterogeneity in ESs across pain threshold studies (other pain outcomes were not examined due to limited data not meeting RVE requirements). Functional state (ON/OFF) was included as an additional variable in each analysis to maximise power by allowing use of all pain threshold data (i.e. from both $\mathrm{ON}$ and OFF states), and given that functional state during testing is likely to represent a substantial source of variation in ES across studies that should be controlled for as a possible confounder. In addition, including functional state as a moderator allows the direct assessment of whether differences across ON vs. OFF states identified in the meta-analysis (Section 3.5.1) were significant.

\subsubsection{PD severity}

We examined whether reduced pain threshold (greater pain sensitivity) in PD was exacerbated in patients with more advanced disease states, by including disease duration and UPDRS-III scores as moderators in separate analyses. Results indicated that differences between PD and controls in pain threshold were not significantly influenced by disease duration $(k=19 ; \mathrm{B}=0.035$, $p=0.24)$ or UPDRS-III symptom severity $(k=19 ; \mathrm{B}=0.006, \mathrm{p}=0.71)$.

Results also indicated that effect size was significantly larger $\left(k=19 ; \Delta S M D=0.33, \mathrm{Cl}_{95}[0.02,0.64], p=0.039\right)$ for $\operatorname{OFF}(\mathrm{B}=0.58)$ than $\mathrm{ON}(\mathrm{B}=0.25)$ states. This pattern of results was reproduced $\left(k=19 ; \Delta S M D=0.33, \mathrm{Cl}_{95}[0.02,0.66], \mathrm{p}=0.041\right)$, when analysis was restricted to $\mathrm{ON}$ states achieved through dopamine medication only (i.e. the single deep brain stimulation study was excluded).

\subsubsection{Method of assessment}

Entering assessment method (limits vs. levels/adjustment) as a moderator in meta-regression of pain threshold revealed that the difference in pain threshold between PD patients and controls was larger when the method of levels/adjustment was used, although this failed to reach statistical significance $(k=18 ; \triangle S M D=0.34$, $p=0.061$ ).

\subsubsection{Secondary moderators}

Separate meta-regression analyses were conducted to explore other moderators specified in Section 2.7.3. As 15 pain threshold studies explicitly stated the use of established diagnostic criteria and 5 studies did not provide information, we also examined this variable as a potential moderator. Given limited data for certain anatomic categories, we collapsed data to form two broad anatomic categories of arm (forearm, upper arm, hand) and leg (leg, foot). For the multiple categorical variable of stimulus modality, a nointercept model was analysed so that each coefficient represented the absolute ES for each stimulus modality (rather than the difference in ES between that modality and an arbitrary reference modality).

No evidence was found for moderating effects of studies' gender composition $(k=20 ; p=0.38)$, mean study age $(k=20$; $p=0.62)$, affected $v s$. non-affected side tested $(k=9 ; p=0.91)$, anatomic site tested $(k=17 ; p=0.43)$ or diagnostic criteria provided $(k=20 ; \mathrm{p}=0.55)$. For stimulus modality $(\mathrm{k}=20)$, however, PD patients demonstrated lower pain threshold than HCs in response to cold $(S M D=0.62, p=0.011)$, electrical $(S M D=0.53, p<0.001)$ and pressure $(S M D=0.41, p=0.055)$ stimuli, but not heat stimuli $(S M D=-0.05, p=0.79)$. PD-related pain could not be reliably assessed as a moderator, as the adjusted $d f$ was <4 (Tipton, 2015).

\subsubsection{Study validity criteria}

Given low endorsement for validity criteria of selection of cases/controls and reporting of pain medication use (Section 3.4), these variables were entered as moderators in separate metaregression analyses of pain threshold. Neither case selection $(p=0.79)$ or control selection $(p=0.68)$ significantly moderated the ES. Differences in pain threshold between PD patients and controls were amplified in studies explicitly stating that participants were not using pain medication, although this did not achieve statistical significance $(\triangle S M D=0.40, p=0.052)$.

\subsection{Repeated-measures studies comparing ON vs. OFF states}

As previous analyses (Section 3.6.1) revealed that differences between PDs and controls were reduced during the ON state, we conducted a stricter evaluation by repeating the analysis including only the 7 studies that directly compared ON $v s$. OFF in a repeatedmeasures designs to ensure superior control of confounds. Results were in line with previous findings (Section 3.6.1), with differences in pain threshold between PD patients and HCs attenuated during ON states $\left(k=7 ; \Delta S M D=-0.25, \mathrm{Cl}_{95}[-0.11,-0.38], p=0.004\right)$. Removal of two studies that did not randomize/counterbalance ON/OFF state or failed to test HCs at equivalent intervals, had little impact on results $(k=5 ; \Delta S M D=-0.22, p=0.045)$. Only 4 studies provided L-DOPA dosages during ON states, which was not sufficient to meet requirements for RVE meta-regression.

\section{Discussion}

The current meta-analysis is the first to examine whether patients with PD exhibit increased pain sensitivity compared to HCs and to investigate potential moderators. Our comprehensive analysis of 26 studies, primarily assessing pain threshold, with a combined total of 739 PD patients and 553 controls yielded 
several key findings: (1) Overall pain threshold was lower in PD patients (indicating greater pain sensitivity) compared to HCs; (2) Suprathreshold pain was lower in PD patients, although this narrowly failed to reach significance; (3) Abnormal pain thresholds in people with $\mathrm{PD}$ during OFF states $(S M D=0.51)$ were significantly diminished, but not completely normalized during ON states $(S M D=0.23)$ produced by dopaminergic medication; (4) Abnormal pain thresholds were not significantly influenced by symptom severity, disease duration, sex or age; (5) While most (16/20) individual studies indicated lower pain threshold in $\mathrm{PD}$, moderate to high variation in effect sizes suggests that other, unidentified variables could influence abnormal pain responses in PD.

\subsection{Pain hypersensitivity in PD and clinical implications}

Results from our meta-analysis provide evidence that patients with PD demonstrate greater sensitivity to noxious stimulation compared to HCs, which seem to be independent of age and sex, and which occur for most types of aversive stimuli. Although no overall differences were found for heat stimulation, the post-hoc nature of this result means any conclusions of modality-specific nociceptive processing abnormalities in PD should be made extremely cautiously and would require independent replication.

Taken together, these results suggest that the increased prevalence and intensity of clinical pain complaints in PD could be, at least in part, influenced by abnormal nociceptive processing. In particular, these results could explain the increased prevalence of non-musculoskeletal pain complaints (e.g., neuropathic pain), which do not obviously directly result from motor dysfunction. Although care must be taken in translating effect sizes from experimentally-induced pain to real-life pain experiences, the fact that an $S M D=0.51$ (in medication-free OFF states) can be classified as a moderate effect (Cohen, 1988), provides some preliminary indication that the impact of PD on pain complaints outside of the laboratory may not be trivial.

There is also some tentative evidence to suggest that the true extent of increased pain sensitivity could be underestimated by the moderate effect size we observed. Studies that explicitly stated that participants were not receiving pain medication (with PD patients generally being more likely to regularly use painkillers) were linked to a larger effect size (an SMD increase by 0.40 ). In addition, studies employing the methods of levels/adjustment, which has been argued to give a more accurate estimate of pain threshold than the method of limits due to a reduced reaction time artefact, also demonstrated a larger effect size (an SMD increase by 0.34 ). It is important that these findings are treated cautiously, as although suggestive, neither subgroup analysis reached statistical significance $(p=0.052-0.061)$.

\subsection{Role of dopamine}

The partial normalisation of the atypical pain thresholds in PD resulting from dopaminergic medication appears to be a robust finding, which was observed when all studies were examined and when only repeated-measures studies, which provide superior control of potential confounds, were included. This partial normalisation of pain threshold has a number theoretical and clinical implications which warrant further elaboration.

First, this finding offers indirect evidence for an underlying role of dopamine depletion in pain hypersensitivity in PD. Although identifying exact underlying mechanisms is difficult from the available data, dopamine could elicit pain hypersensitivity either indirectly through modulatory effects on affective pain processing and/or directly by affecting neuronal activity at key pain-modulating areas in the brain such as the thalamus, basal ganglia, insula, anterior cingulate cortex and periaqueductal grey
(Fil et al., 2013; Jarcho et al., 2012). Reduced dopaminergic neurotransmission may impair natural analgesia through a decreased activation of dopamine-mediated pain inhibitory pathways. These descend from the substantia nigra to the substantia gelatinosa in the spinal cord and inhibit transmission of ascending nociceptive signals (Fil et al., 2013). This direct role of dopamine is consistent with PET studies in healthy participants that show an association between greater subjective pain and decreased dopamine activity (Cury et al., 2016), the diminished pain response seen in schizophrenia (Stubbs et al., 2015) a disorder linked to aberrant dopaminergic neurotransmission, and evidence from animal models suggesting a role of dopamine in chronic regional pain syndrome (Wood, 2008). Experimental research on pain in PD patients assessing the effect of pro-dopaminergic and antidopaminergic states or medications could help further elucidate the role of dopamine in pain perception.

Second, if attenuation of pain can be achieved through dopaminergic medication, this suggests that the development of classes of novel compounds that efficiently target dopamine pain-inhibitory pathways may have potential as effective analgesics. Such compounds may be effective analgesics both for PD and for other painful disorders linked to disrupted endogenous dopamine activity such as fibromyalgia, burning mouth syndrome and painful diabetic neuropathy (Jarcho et al., 2012; Wood, 2008). Furthermore, if the suggestion that the underuse of conventional painkillers in PD patients is attributable to poor efficacy in this group (Perez-Lloret et al., 2012), such medications could provide potentially superior alternatives. The utility of such analgesics could even extend to conventional treatment in healthy individuals with pain. While the role of opioids and non-dopaminergic (e.g. noradrenergic or serotoninergic) pathways is well recognised, the current findings further suggest a meaningful role for dopamine-mediated analgesia and may provide the impetus for further study involving preclinical models and neuroimaging techniques in humans.

\subsection{Partial pain threshold normalisation}

The fact that dopamine medication diminished but did not appear to completely normalise pain threshold, suggests that additional mechanisms are likely to contribute to pain hypersensitivity in PD. Although it is difficult to identify such mechanisms from the current available data, these could include a loss of epidermal nerve fibres (Nolano et al., 2008) or deficiencies in other, nondopaminergic pain pathways. An alternative explanation is that pain hypersensitivity is entirely mediated by dopamine deficiency in PD but that study medication dosages were insufficient to restore dopamine neurotransmission to normal physiological levels. Due to insufficient available data, we were not able to assess whether higher L-DOPA dosages were associated with greater normalisation of pain threshold.

\subsection{Independence of motor impairment and pain}

One notable finding was that pain sensitivity did not increase with increasing disease severity or duration. While this contrasts with clinical surveys which have shown the frequency and intensity of pain to be greater in advanced-stage compared to early-stage PD (Valkovic et al., 2015), this research refers primarily to musculoskeletal pain. While disease progression would be expected to intensify this type of pain due to increasing muscle rigidity or postural abnormalities (Cury et al., 2016), our findings suggest that changes in sensitivity to noxious input may occur early on and be relatively independent of motor function degradation. 


\subsection{Limitations}

Whilst these data provide novel insights into altered pain perception in PD, some limitations should be noted. Firstly, findings are based primarily on pain threshold which was the most commonly studied pain outcome. Although an established measure of pain sensitivity, pain threshold represents pain at the lower end of the intensity continuum and cannot be automatically generalised to more intense levels of clinical pain. Second, although adequate data was available for meta-regression, genuine moderating effects of variables (e.g. disease duration) that were non-significant cannot be dismissed, and may be detectable with more available data; especially if the magnitude of these effects is small. Third, although exclusion of PD patients with cognitive impairment in primary studies was necessary to maximise adherence to experimental requirements, current findings should be necessarily restricted to PD patients who have relatively preserved overall cognition (Folstein et al., 1975).

\subsection{Future research directions}

Despite these limitations, the current findings provide strong support that individuals with PD exhibit greater sensitivity to noxious stimuli than HCs, based on laboratory studies that provide a control of potential confounders not easily achievable in clinical settings. Future studies are needed to help establish whether pain hypersensitivity in PD extends to suprathreshold levels of pain, and insights may also be gained from the use of ischemic and dermal capsaicin experimental pain models that evoke several aspects of chronic pain whilst preserving strict experimental control (Staahl et al., 2009). More research involving preclinical models and neuroimaging techniques in humans would also help to elucidate potential mechanistic pathways underlying altered pain perception in PD.

\subsection{Conclusions}

To the authors' knowledge, this is the only published metaanalysis of studies comparing PD patients with healthy controls in their response to controlled experimental pain stimulation. Results indicate significantly lower pain threshold in PD patients (indicative of greater pain) in the OFF state compared to controls. This was partially (but not completely) normalized by dopaminergic medication, suggesting that disruption of dopamine pain pathways may contribute to abnormal pain processing. These findings suggest that PD may confer a hypersensitivity to nociceptive information that could both exacerbate the musculoskeletal pain resulting from motor rigidity and abnormal posture control, and contribute to the less common but nevertheless troubling non-musculoskeletal pain complaints that occur in PD.

\section{Disclosures}

TT, KG, NV, EW and BS have no conflicts of interest. AFC is supported by a research fellowship award from the Conselho Nacional de Desenvolvimento Científico e Tecnológico (CNPq; Brazil). CUC has been a consultant and/or advisor to or has received honoraria from: Alkermes, Allergan, Bristol-Myers Squibb, Forum, Gerson Lehrman Group, IntraCellular Therapies, Janssen/J\&J, LB Pharma, Lundbeck, Medavante, Medscape, Neurocrine, Otsuka, Pfizer, ProPhase, Sunovion, Supernus, Takeda, and Teva. He has provided expert testimony for Bristol-Myers Squibb, Janssen, and Otsuka. He served on a Data Safety Monitoring Board for Lundbeck and Pfizer. He received grant support from Takeda.

\section{Acknowledgements}

We are especially grateful to Drs Gergely Orsi, Yelena Granovsky, Jon Stoessl, Maria Nolano, Tomohiko Nakamura, Masanaka Takeda, Janosch Priebe for their rapid and exceptionally helpful responses to requests for additional study data. We are also extremely grateful to Professor John Lees and Dr Alastair Noyce of University College London for their invaluable advice and extremely helpful commentary on the manuscript.

\section{Appendix A. Supplementary data}

Supplementary data associated with this article can be found, in the online version, at http://dx.doi.org/10.1016/j.arr.2017.01.005.

\section{References}

Allen, N.E., Moloney, N., Hassett, L.M., Canning, C.G., Lewis, S.J.G., Cruz-Mavignier K., Barry, B.K., 2016. Exercise induced analgesia is present in people with Parkinson's disease. Mov. Disord. 31.

Apkarian, A.V., Bushnell, M.C., Treede, R.D., Zubieta, J.K., 2005. Human brain mechanisms of pain perception and regulation in health and disease. Eur. J. Pain 9, 463-484.

Aschermann, Z., Nagy, F., Perlaki, G., Janszky, J., Schwarcz, A., Kovacs, N., Bogner, P., Komoly, S., Orsi, G., 2015. Wind-up' in Parkinson's disease: a functional magnetic resonance imaging study. Eur. J. Pain 19, 1288-1297.

Borenstein, M., Hedges, L., Higgins, J., Rothstein, H.R., 2009. Introduction to Meta-Analysis. Wiley, West Sussex.

Brefel-Courbon, C., Payoux, P., Thalamas, C., Ory, F., Quelven, I., Chollet, F., Montastruc, J.L., Rascol, O., 2005. Effect of levodopa on pain threshold in Parkinson's disease: a clinical and positron emission tomography study. Mov. Disord. 20, 1557-1563.

Broen, M.P., Braaksma, M.M., Patijn, J., Weber, W.E., 2012. Prevalence of pain in Parkinson's disease: a systematic review using the modified QUADAS tool. Mov. Disord. 27, 480-484

Chaudhuri, K.R., Odin, P., 2010. The challenge of non-motor symptoms in Parkinson's disease. Prog. Brain Res. 184, 325-341.

Chaudhuri, K.R., Schapira, A.H., 2009. Non-motor symptoms of Parkinson's disease: dopaminergic pathophysiology and treatment. Lancet Neurol. 8, 464-474.

Chaudhuri, K.R., Prieto-Jurcynska, C., Naidu, Y., Mitra, T., Frades-Payo, B., Tluk, S., Ruessmann, A., Odin, P., Macphee, G., Stocchi, F., Ondo, W., Sethi, K., Schapira, A.H., Martinez Castrillo, J.C., Martinez-Martin, P., 2010. The nondeclaration of nonmotor symptoms of Parkinson's disease to health care professionals: an international study using the nonmotor symptoms questionnaire. Mov. Disord. 25, 704-709.

Chen, Y., Mao, C.J., Li, S.J., Wang, F., Chen, J., Zhang, H.J., Li, L., Guo, S.S., Yang, Y.P., Liu, C.F., 2015. Quantitative and fiber-selective evaluation of pain and sensory dysfunction in patients with Parkinson's disease. Parkinsonism Relat. Disord. 21, 361-365.

Ciampi de Andrade, D., Lefaucheur, J.P., Galhardoni, R., Ferreira, K.S., Brandão Paiva, A.R., Bor-Seng-Shu, E., Alvarenga, L., Myczkowski, M.L., Marcolin, M.A., de Siqueira, S.R., Fonoff, E., Barbosa, E.R., Teixeira, M.J., 2012. Subthalamic deep brain stimulation modulates small fiber-dependent sensory thresholds in Parkinson's disease. Pain 153, 1107-1113.

Cohen, J., 1988. Statistical Power Analysis for the Behavioral Sciences. Lawrence Earlbaum Associates, Hillsdale, NJ.

Cury, R.G., Galhardoni, R., Fonoff, E.T., Perez Lloret, S., Dos Santos Ghilardi, M.G. Barbosa, E.R., Teixeira, M.J., Ciampi de Andrade, D., 2016. Sensory abnormalities and pain in Parkinson disease and its modulation by treatment of motor symptoms. Eur. J. Pain 20, 151-165.

Deeks, J.J., Dinnes, J., D’Amico, R., Sowden, A.J., Sakarovitch, C., Song, F., Petticrew, M., Altman, D.G., International, S.T.C.G., European, C.S.T.C.G., 2003. Evaluating non-randomised intervention studies. Health Technol. Assess. 7, iii-X, 1.

Djaldetti, R., Shifrin, A., Rogowski, Z., Sprecher, E., Melamed, E., Yarnitsky, D., 2004. Quantitative measurement of pain sensation in patients with Parkinson disease. Neurology 62, 2171-2175.

Duval, S., Tweedie, R., 2000. Trim and fill: a simple funnel-plot-based method of testing and adjusting for publication bias in meta-analysis. Biometrics 56 455-463.

Egger, M., Davey Smith, G., Schneider, M., Minder, C., 1997. Bias in meta-analysis detected by a simple, graphical test. BMJ 315, 629-634

Fahn, S., Elton, R.L., 1987. In: Fahn, S., Marsden, C.D., Calne, D.B., Goldstein, M. (Eds.), Recent Developments in Parkinson's Disease II. MacMillan Health Care Information, New Jersey, pp. 153-163.

Fil, A., Cano-de-la-Cuerda, R., Muñoz-Hellín, E., Vela, L., Ramiro-González, M., Fernández-de-Las-Peñas, C., 2013. Pain in Parkinson disease: a review of the literature. Parkinsonism Relat. Disord. 19, 285-294, discussion 285.

Fisher, Z., Tipton, E., 2014. Robumeta: Robust variance meta-regression. R package version 1.3. 
Folstein, M.F., Folstein, S.E., McHugh, P.R., 1975. Mini-mental state: a practical method for grading the cognitive state of patients for the clinician. J. Psychiatr. Res. 12, 189-198.

Ford, B., 2010. Pain in parkinson's disease. Mov. Disord. 25 (Suppl. 1), S98-103.

Gerdelat-Mas, A., Simonetta-Moreau, M., Thalamas, C., Ory-Magne, F., Slaoui, T., Rascol, O., Brefel-Courbon, C., 2007. Levodopa raises objective pain threshold in Parkinson's disease: a RIII reflex study. J. Neurol. Neurosurg. Psychiatry 78, $1140-1142$.

Goetz, C.G., Stebbins, G.T., Tilley, B.C., 2012. Calibration of unified Parkinson's disease rating scale scores to Movement Disorder Society-unified Parkinson's disease rating scale scores. Mov. Disord. 27, 1239-1242.

Granovsky, Y., Schlesinger, I., Fadel, S., Erikh, I., Sprecher, E., Yarnitsky, D., 2013. Asymmetric pain processing in Parkinson's disease. Eur. J. Neurol. 20, 1375-1382.

Grashorn, W., Schunke, O., Buhmann, C., Forkmann, K., Diedrich, S., Wesemann, K., Bingel, U., 2015. Influence of dopaminergic medication on conditioned pain modulation in parkinson's disease patients. PLoS One 10, e0135287.

Gwet, K., 2002. Inter-rater reliability: dependency on trait prevalence and marginal homogeneity. Stat. Methods Inter-Rater Reliab. Assessment 2, 1-9.

Hara, T., Hirayama, M., Mizutani, Y., Hama, T., Hori, N., Nakamura, T., Kato, S., Watanabe, H., Sobue, G., 2013. Impaired pain processing in Parkinson's disease and its relative association with the sense of smell. Parkinsonism Relat. Disord. 19, 43-46.

Hedges, L.V., Tipton, E., Johnson, M.C., 2010. Robust variance estimation in meta-regression with dependent effect size estimates. Res. Synth. Methods 1, 39-65.

Higgins, J.P., Thompson, S.G., Deeks, J.J., Altman, D.G., 2003. Measuring inconsistency in meta-analyses. BMJ 327, 557-560.

Jarcho, J.M., Mayer, E.A., Jiang, Z.K., Feier, N.A., London, E.D., 2012. Pain, affective symptoms, and cognitive deficits in patients with cerebral dopamine dysfunction. Pain 153, 744-754.

Lim, S.-Y., Farrell, M.J., Gibson, S.J., Helme, R.D., Lang, A.E., Evans, A.H., 2008. Do dyskinesia and pain share common pathophysiological mechanisms in Parkinson's disease. Mov. Disord. 23, 1689-1695.

Lipsey, M., Wilson, D., 2001. Practical Meta-Analysis. Sage, London.

Maruo, T., Saitoh, Y., Hosomi, K., Kishima, H., Shimokawa, T., Hirata, M., Goto, T., Morris, S., Harada, Y., Yanagisawa, T., Aly, M.M., Yoshimine, T., 2011. Deep brain stimulation of the subthalamic nucleus improves temperature sensation in patients with Parkinson's disease. Pain 152, 860-865.

Massetani, R., Lucchetti, R., Vignocchi, G., Siciliano, G., Rossi, B., 1989. Pain threshold and polysynaptic components of the blink reflex in Parkinson's disease. Funct. Neurol. 4, 199-202.

Mitra, T., Naidu, Y., Martinez-Martin, P., 2008. The non declaration of non motor symptoms of Parkinson's disease to healthcare professionals. An international survey using the NMSQuest. In: 6th International Congress on Mental Dysfunctions and Other Non-motor Features in Parkinson's Disease and Related Disorders Dresden October, 2008 Park Related Disorders, P0II:161.

Moher, D., Liberati, A., Tetzlaff, J., Altman, D.G., PRISMA, G., 2009. Preferred reporting items for systematic reviews and meta-analyses: the PRISMA statement. BMJ 339, b2535.

Mylius, V., Engau, I., Teepker, M., Stiasny-Kolster, K., Schepelmann, K., Oertel, W.H., Lautenbacher, S., Möller, J.C., 2009. Pain sensitivity and descending inhibition of pain in Parkinson's disease. J. Neurol. Neurosurg. Psychiatry 80, 24-28.

Mylius, V., Brebbermann, J., Dohmann, H., Engau, I., Oertel, W.H., Möller, J.C., 2011 Pain sensitivity and clinical progression in Parkinson's disease. Mov. Disord. 26, 2220-2225.

Mylius, V., Pee, S., Pape, H., Teepker, M., Stamelou, M., Eggert, K., Lefaucheur, J.P., Oertel, W.H., Möller, J.C., 2016. Experimental pain sensitivity in multiple system atrophy and Parkinson's disease at an early stage. Eur. J. Pain 20, 1223-1228.

Nègre-Pagès, L., Regragui, W., Bouhassira, D., Grandjean, H., Rascol, O., DoPaMiP, S.G., 2008. Chronic pain in Parkinson's disease: the cross-sectional French DoPaMiP survey. Mov. Disord. 23, 1361-1369.

Nandhagopal, R., Troiano, A.R., Mak, E., Schulzer, M., Bushnell, M.C., Stoessl, A.J., 2010. Response to heat pain stimulation in idiopathic Parkinson's disease. Pain Med. 11, 834-840.

Nolano, M., Provitera, V., Estraneo, A., Selim, M.M., Caporaso, G., Stancanelli, A., Saltalamacchia, A.M., Lanzillo, B., Santoro, L., 2008. Sensory deficit in Parkinson's disease: evidence of a cutaneous denervation. Brain 131, 1903-1911.
Perez-Lloret, S., Rey, M.V., Dellapina, E., Pellaprat, J., Brefel-Courbon, C., Rascol, O., 2012. Emerging analgesic drugs for Parkinson's disease. Expert Opin. Emerg. Drugs 17, 157-171.

Priebe, J.A., Kunz, M., Morcinek, C., Rieckmann, P., Lautenbacher, S., 2016 Electrophysiological assessment of nociception in patients with Parkinson's disease: a multi-methods approach. J. Neurol. Sci. 368, 59-69.

Quittenbaum, B.H., Grahn, B., 2004. Quality of life and pain in Parkinson's disease: a controlled cross-sectional study. Parkinsonism Relat. Disord. 10, 129-136.

R Core Team, 2014. R: A Language and Environment for Statistical Computing. R Foundation for Statistical Computing, Vienna, Austria.

Schestatsky, P., Kumru, H., Valls-Sole, J., Valldeoriola, F., Marti, M.J., Tolosa, E., Chaves, M.L., 2007. Neurophysiologic study of central pain in patients with Parkinson disease. Neurology 69, 2162-2169.

Schrag, A., Horsfall, L., Walters, K., Noyce, A., Petersen, I., 2015. Prediagnostic presentations of Parkinson's disease in primary care: a case-control study. Lancet Neurol. 14, 57-64.

Staahl, C., Olesen, A.E., Andresen, T., Arendt-Nielsen, L., Drewes, A.M., 2009. Assessing analgesic actions of opioids by experimental pain models in healthy volunteers - an updated review. Br. J. Clin. Pharmacol. 68, 149-168.

Stamelou, M., Dohmann, H., Brebermann, J., Boura, E., Oertel, W.H., Höglinger, G., Möller, J.C., Mylius, V., 2012. Clinical pain and experimental pain sensitivity in progressive supranuclear palsy. Parkinsonism Relat. Disord. 18, 606-608.

Stroup, D.F., Berlin, J.A., Morton, S.C., Olkin, I., Williamson, G.D., Rennie, D., Moher, D., Becker, B.J., Sipe, T.A., Thacker, S.B., 2000. Meta-analysis of observational studies in epidemiology: a proposal for reporting. Meta-analysis of Observational Studies in Epidemiology (MOOSE) group. JAMA 283, 2008-2012.

Stubbs, B., Thompson, T., Acaster, S., Vancampfort, D., Gaughran, F., Correll, C.U., 2015. Decreased pain sensitivity among people with schizophrenia: a meta-analysis of experimental pain induction studies. Pain 156, 2121-2131.

Takeda, M., Okada, F., Tachibana, H., Kajiyama, K., Yoshikawa, H., 2013. Neurophysiologic study of pain in Parkinson's disease: a study with pain-related evoked potentials. Mov. Disord. 28, S79.

Takeda, M., Tachibana, H., Okada, F., Kasama, S., Yoshikawa, H., 2014. Pain in patients with parkinson's disease; a pain-related evoked potential study. Neurosci. Biomed. Eng. 2, 36-40.

Tan, Y., Tan, J., Luo, C., Cui, W., He, H., Bin, Y., Deng, J., Tan, R., Tan, W., Liu, T., Zeng, N., Xiao, R., Yao, D., Wang, X., 2015. Altered brain activation in early drug-naive Parkinson's disease during heat pain stimuli: an fMRI study. Parkinson's Dis. 2015, 273019.

Thompson, T., Correll, C.U., Gallop, K., Vancampfort, D., Stubbs, B., 2016. Is pain perception altered in people with depression? A systematic review and meta-analysis of experimental pain research. J. Pain 17, 1257-1272.

Tipton, E., 2015. Small sample adjustments for robust variance estimation with meta-regression. Psychol. Methods 20, 375-393.

Valkovic, P., Minar, M., Singliarova, H., Harsany, J., Hanakova, M., Martinkova, J., Benetin, J., 2015. Pain in parkinson's disease: a cross-sectional study of its prevalence, types, and relationship to depression and quality of life. PLoS One 10, e0136541.

Vela, L., Lyons, K.E., Singer, C., Lieberman, A.N., 2007. Pain-pressure threshold in patients with Parkinson's disease with and without dyskinesia. Parkinsonism Relat. Disord. 13, 189-192.

Vela, L., Cano-de-la-Cuerda, R., Fil, A., Muñoz-Hellín, E., Ortíz-Gutiérrez, R., Macías-Macías, Y., Fernández-de-Las-Peñas, C., 2012. Thermal and mechanical pain thresholds in patients with fluctuating Parkinson's disease. Parkinsonism Relat. Disord. 18, 953-957.

Wells, G.A., Shea, B., O'Connell, D., Peterson, J., Welch, V., Losos, M., Tugwell, P., 2008. The Newcastle-Ottawa Scale (NOS) for Assessing the Quality of Nonrandomised Studies in Meta-Analyses (Accessed 8 May 2016). Available at: http://www.ohri.ca/programs/clinical_epidemiology/oxford.asp.

Wood, P.B., 2008. Role of central dopamine in pain and analgesia. Expert Rev. Neurother. 8, 781-797.

Yarnitsky, D., Pud, D., 2004. In: Binnie, C.D., Cooper, R., Mauguiere, F., Osselton, J.W., Prior, P.F., Tedman, B.M. (Eds.), Clinical Neurophysiology: EMG, Nerve Conduction and Evoked Potentials, vol. 1. Elsevier, Amsterdam, p. 305

Zambito, M.S., Tinazzi, M., Vitaliani, R., Recchia, S., Fabris, F., Marchini, C., Fiaschi, A., Moretto, G., Giometto, B., Macerollo, A., Defazio, G., 2011. Spontaneous pain, pain threshold, and pain tolerance in Parkinson's disease. J. Neurol. 258, 627-633. 